\title{
ON THE ESTIMATES OF THE ALGEBRAIC INDEPENDENCE MEASURES OF THE VALUES OF E-FUNCTIONS
}

\author{
A. B. SHIDLOVSKII \\ Dedicated to K. Mahler on his 75th birthday
}

(Received 6 July 1978)

Communicated by J. H. Coates

\begin{abstract}
The work establishes the estimates of linear independence, transcendence, and algebraic independence measures of the values at algebraic points of a set of $E$-functions, satisfying linear differential equations with coefficients from $\mathbf{C}(z)$, rather close to their natural bounds.
\end{abstract}

Subject classification (Amer. Math. Soc. (MOS) 1970): 10 F 35.

\section{Notations and Definitions}

The work establishes the estimates of linear independence, transcendence, and algebraic independence measures of the values at algebraic points of a set of $E$ functions, satisfying linear differential equations with coefficients from $C(z)$, rather close to their natural bounds. This history of the subject is given in [1], [2] and [3].

In what follows $\mathbf{K}$ denotes an algebraic number field over $\mathbf{Q}$ of degree $h$ and $\mathbf{I}$ some imaginary quadratic field. If $\alpha \in \mathbf{K}$, then $\alpha^{*}=\max _{1 \leqslant i \leqslant h}\left|\alpha_{i}\right|$, where $\alpha_{1}, \ldots, \alpha_{h}$ are the conjugates to $\alpha$ in $\mathbf{K}$.

The algebraic independence measure of numbers $\xi_{1}, \ldots, \xi_{m}$ is the function

$$
\varphi\left(\xi_{1}, \ldots, \xi_{m} ; n_{1}, \ldots, n_{m} ; H\right)=\min \left|P\left(\xi_{1}, \ldots, \xi\right)\right|,
$$

where $P=P\left(z_{1}, \ldots, z_{m}\right) \in \mathrm{Z}\left[z_{1}, \ldots, z_{m}\right], P \neq \equiv, H_{P} \leqslant H, H_{P}$ is the height of $P$ and the (C) Copyright Australian Mathematical Society 1979

Copyright. Apart from any fair dealing for scholarly purposes as permitted under the Copyright Act, no part of this JOURNAL may be reproduced by any process without written permission from the Treasurer of the Australian Mathematical Society. 
degree of $P$ in $z_{1}, \ldots, z_{m}$ is less than or equal to $n_{1}, \ldots, n_{m}$ correspondingly, and the maximum is considered over all the polynomials, satisfying these conditions.

If $m=1$ then the function

$$
\varphi(\xi ; n ; H)=\min |P(\xi)|
$$

is called the transcendence measure of number $\xi$.

If $n_{k}=1, k=1, \ldots, m$, then (in homogeneous case) the function

$$
L\left(\xi_{1}, \ldots, \xi_{m} ; H\right)=\min \left|a_{1} \xi_{1}+\ldots+a_{m} \xi_{m}\right|,
$$

where $a_{k} \in \mathbf{Z},\left|a_{k}\right| \leqslant H, k=1, \ldots, m, a_{1}^{2}+\ldots+a_{m}^{2}>0$, is called the linear independence measure of numbers $\xi_{1}, \ldots, \xi_{m}$ and the function $L(1 ; \xi ; H)$ is the irrationality measure of $\xi$.

We often consider as the measure of algebraic independence the function

$$
\varphi\left(\xi_{1}, \ldots, \xi_{m} ; n ; H\right)
$$

which is defined in an analogous way, but where the degree in $z_{1}, \ldots, z_{m}$ does not exceed $n$, and in some cases the function

$$
\varphi\left(\xi_{1,1}, \ldots, \xi_{m, 1} ; \ldots ; \xi_{1, s}, \ldots, \xi_{m_{s}, s} ; n_{1}, \ldots, n_{s} ; H\right),
$$

which is defined in a similar way but where the set of the given numbers consists of $s$ groups and the numbers $n_{1}, \ldots, n_{s}$ are the bounds of the degrees of $P$ in the corresponding groups of variables.

With the help of Dirichlet principle one can easily obtain the upper estimates of measures for any sets of numbers $\xi$ (see, for example, [1] and [3]). In the theory of transcendental numbers and its applications it is of interest to obtain the lower estimates of measures for some classes of numbers $\xi$.

If we know the lower estimate, for example, for $\varphi(\xi ; n ; H)$, then this estimate holds for $|P(\xi)|$, where $P(z) \in \mathbf{Z}[z], P(z) \neq \equiv 0, \operatorname{deg} P \leqslant n, H_{P} \leqslant H$.

Siegel (see [4] and [5]) calls an entire function

$$
f(z)=\sum_{n=0}^{\infty} c_{n} \frac{z_{n}}{n !}
$$

an $E$-function, if

(1) $c_{n} \in \mathbf{K}, n=0,1, \ldots$;

(2) for any $\varepsilon>0, c_{n}^{*}=O\left(n^{\epsilon n}\right)$, when $n \rightarrow \infty$;

(3) there exists a sequence $\left\{q_{n}\right\}, q_{n} \in \mathbf{N}$, such that the numbers $q_{n} c_{k} \in \mathbf{Z}_{\mathbf{k}}$, $k=0,1, \ldots, n$, for all $n=0,1, \ldots$, and for any $\varepsilon>0, q_{n}=O\left(n^{\epsilon n}\right), n \rightarrow \infty$.

The $E$-functions (6) with the coefficients of their power series from the field $\mathbf{K}$ will be called $K E$-functions.

In papers [3] and [6] $I E$-functions are considered which satisfy linear differential equations with coefficients from $\mathbf{C}(z)$. For the set of values of such functions at 
the points from I, rather precise lower estimates for linear independence, transcendence and algebraic independence measures are obtained.

In this work after some changes in the definition of the $E$-function, the theorems of papers [3] and [6] are essentially refined at the expense of the refinement of the remainder term of the exponent. For the proof we use Siegel's well-known method and its generalization, given in [7]. The most important lemmas of the method are refined like that that is made in [8] but the main parameter, the order of zero at $z=0$ of the basic approximating form, is selected in an optimal way.

Besides, in the estimates of the transcendence measures in this paper, the degree of the polynomial may enlarge together with the height up to some limit. A part of the results of this paper is formulated without proof in [9] in a less precise form. In paper [9] it was shown for the first time that it is possible to enlarge the degree together with the height in the estimates of measures, obtained with the considered method. Later a similar result in another case was established by Nesterenko [10]. In the proofs of Theorems 3, 4 and 5 of this work we use the estimate of the order of zero at $z=0$ of a polynomial of $z$ and the considered functions, obtained in [10]. For absolute understanding of the subject matter of this paper it is necessary to get acquainted with the works [3], [7] and [8].

We generalize the earlier introduced notions of measures and consider the algebraic independence, transcendence and linear independence measures with respect to $\mathbf{K}$, which are defined just like (1)-(5) with the only difference being that the coefficients of the corresponding polynomial $P$ or the linear form $L$ belong to $\mathbf{Z}_{\mathbf{K}}$ and the maximum of the moduli of the coefficients of $P$ or of the linear form $L$ and of all their conjugates do not exceed $H$. The corresponding measures will be denoted analogously to (1)-(5) but instead of the symbols $\varphi$ and $L$ we write $\varphi_{\mathbf{K}}$ and $L_{\mathbf{K}}$.

Lastly, let us consider the homogeneous algebraic independence measures $\varphi^{0}\left(\xi_{1}, \ldots, \xi_{m} ; n ; H\right)$ and $\varphi_{\mathrm{K}}^{0}\left(\xi_{1}, \ldots, \xi_{m} ; n ; H\right)$, which are defined by analogy with (4) with the only difference being that in their definitions $P\left(z_{1}, \ldots, z_{m}\right)$ is a homogeneous polynomial of degree not greater than $n$, correspondingly from $\mathbf{Z}\left[z_{1}, \ldots, z_{m}\right]$ or $\mathbf{Z}_{\mathbf{K}}\left[z_{1}, \ldots, z_{m}\right]$.

It is evident that on establishing the estimates of the introduced measures we have obtained the estimates for the values of polynomials in $\mathbf{Z}_{\mathbf{K}}\left[z_{1}, \ldots, z_{m}\right]$ with corresponding degrees and heights.

Let us change the definition of the $E$-function in the following way. We call the entire function (6) the $E$-function, if it satisfies three conditions analogous to the conditions of the original definition but in which the estimates of $c_{n}$ and $q_{n}$ are replaced correspondingly by

$$
c_{n}^{*}=O\left(c^{n}\right), \quad q_{n}=O\left(c^{n}\right),
$$

where $c \geqslant 1$ is a fixed number. 
It is easy to verify that the set of all $E$-functions as well as in the case of the original definition forms a ring of functions closed relative to differentiation, integration from 0 to $z$ and substitution $z$ for $\lambda z$ where $\lambda$ is an algebraic number. Note that all the known $E$-functions, satisfying linear differential equations with coefficients in $C(z)$ are $E$-functions in the sense of the new definition.

To make clear the character of the dependence of the constants involved in the definitions of the measures on the considered functions and numbers $\xi, m$ and $n$, we refine the definition of the $E$-function. We shall say that the entire function (6) belongs to the class $K E(\lambda, c ; \mu, q)$ if

(1) $c_{n} \in \mathbf{K}, n=0,1, \ldots$;

(2) there exist constants $\lambda \geqslant 1$ and $c \geqslant 1$ such that $c_{n}^{*} \leqslant \lambda n^{n}, n=0,1, \ldots$;

(3) there exists a sequence $\left\{q_{n}\right\}, q_{n} \in \mathbf{N}$, and constants $\mu \geqslant 1, q \geqslant 1$, such that $q_{n} c_{k} \in \mathbf{Z}_{\mathbf{K}}, k=0,1, \ldots, n$, for all $n=0,1, \ldots$, and $q_{n} \leqslant \mu q^{n}, n=0,1, \ldots$.

Consider a set of functions

$$
f_{k}(z)=\sum_{n=0}^{\infty} \frac{c_{k, n}}{n !}, z^{n}, \quad k=1, \ldots, m,
$$

from the class $K E(\lambda, c ; \mu, q)$. Then there exist sequences

$$
\left\{q_{0, n}\right\}, \quad q_{0, n} \leqslant \mu_{0} q_{0}^{n}, \quad \mu_{0} \geqslant \mu, \quad q_{0} \geqslant q, \quad k=1, \ldots, m,
$$

such that

$$
q_{0, n} c_{k, v} \in \mathbf{Z}_{\mathbf{K}}, \quad v=0,1, \ldots, n, \quad k=1, \ldots, m .
$$

Indeed, for example, we can take the sequence

$$
q_{0, n}=q_{1, n} \ldots q_{m, n}, \quad n=0,1, \ldots m,
$$

where $\left\{q_{k, n}\right\}, k=1, \ldots, m$, are the sequences thet correspond by definition to each of the $K E$-functions (7). Then it is evident that

$$
\mu_{0} \leqslant \mu^{m}, \quad q_{0}=q^{m}
$$

In some cases there may exist sequences of the form (8) for which $\mu_{0}<\mu^{m}$ and $q_{0}<q^{m}$ as, for example, in the case of Lemma 6 given below.

Later on, for the set of $K E$-functions (7) the numbers $\mu_{0}$ and $q_{0}$ will have the sense determined above.

If $\xi \in \mathbf{K}$ we denote the conjugates to a number $\xi$ in $\mathbf{K}$ by $\xi_{1}, \ldots, \xi_{h}$. We denote by $\mathbf{K}_{i}, i=1, \ldots, h$, the algebraic number fields, conjugate to $\mathbf{K}$ and by $f_{1, i}(z), \ldots, f_{m, i}(z), i=1, \ldots, h$, the $K_{i} E$-functions obtained from the functions ( 7 ) by replacing all the numbers $c_{k, v}$ by their conjugates from $\mathbf{K}_{i}$ and call them the functions, conjugate to functions (7). If a set of $K E$-functions consists of several groups and is numerated by two indices then the conjugate functions will be denoted by the third index. Similar notations for conjugate functions will take place for the set of $K E$-functions, denoted by letter $\Phi$ with indices. 
In future we will consider the sets of $K E$-functions (7) satisfying the system of linear differential equations

$$
y_{k}^{\prime}=Q_{k, 0}+\sum_{i=1}^{m} Q_{k, i} y_{i}, \quad k=1, \ldots, m, \quad Q_{k, i} \in \mathbf{C}(z),
$$

or the system of homogeneous equations

$$
y_{k}^{\prime}=\sum_{i=1}^{m} Q_{k, i} y_{i}, \quad k=1, \ldots, m, \quad Q_{k, i} \in \mathbf{C}(z) .
$$

In this case (see [7]) the numerical coefficients of the polynomials are the numerators and denominators of all the functions $Q_{k, i}$ in (12) or (13) may be selected from $\mathbf{Z}_{\mathbf{K}}[z]$. Thus $Q_{k, i} \in \mathbf{K}(z)$.

Denote by $T=T(z) \in Z_{\mathrm{K}}[z]$ and $T^{0}=T^{0}(z) \in Z_{\mathrm{K}}[z]$ the polynomials which are the least common denominators of all the rational functions $Q_{k, i}$ in (12) and (13). Thus all $T Q_{k, i} \in \mathbf{Z}_{\mathbf{K}}[z]$ and, accordingly, all $T^{\circ} Q_{k, i} \in \mathbf{Z}_{\mathbf{K}}[z]$.

Denote by $g$ the largest of the degrees of $m^{2}+1$ polynomials $T^{0}$ and $T^{0} Q_{k, i}$, $k, i=1, \ldots, m$, and by $G$ the largest of the moduli of the coefficients and their conjugates of these polynomials. The letter $p$ denotes the smallest of the orders of zero at $z=0$ of the $K E$-functions (7) which are under consideration.

If the considered set of functions splits into $s$ groups and each of them satisfies its own system of equation (12) or (13), then $T_{l}=T_{l}(z)$ or $T_{l}^{0}=T_{l}^{0}(z), l=1, \ldots, s$, have just the same sense that $T$ and $T^{0}$ have for systems (12) and (13).

Denote in what follows by the letter $\sigma$, and by the same letter with various indices, positive constants, which depend only on the class $\mathrm{K} E(\lambda, c ; \mu ; q)$, which contains the considered $E$-functions that is only on the field $\mathbf{K}$ and the numbers $\lambda, c, \mu$ and $q$. By the letter $\gamma$ and by the same letter with various indices we denote positive constants also depending on $\operatorname{K} E(\lambda, c ; \mu, q)$, numbers $p, q, G$ and the number $\xi \in \mathbf{K}$.

Note that the numbers $\sigma, \gamma, \sigma_{k}$ and $\gamma_{k}$ do not depend on $m$ (the number of the considered functions) and on the degrees of the polynomials considered below. The positive constants $\delta$ will depend only on the system of differential equations which are satisfied by the considered functions. The constants $\delta$ may depend on $m$, generally speaking. The positive constants $\Omega(m)$ and $\Omega_{1}(m)$ will depend only on the class $\operatorname{K} E(\lambda, c ; \mu, q)$, the system of differential equations, which is satisfied by the considered functions and the numbers $p, q, G, \xi$ and $m$.

All the constants $\sigma, \gamma, \sigma_{k}$ and $\gamma_{k}$ are effective, that is, they may be calculated for any concrete set of $K E$-functions and the constants $\delta$, and $\Omega(m)$ and $\Omega_{1}(m)$ are not effective generally speaking. In different cases the same letter will denote different constants. In this paper square brackets are used for denoting the largest integer in the considered numbers.

We call an irreducible set of functions (see [11]) a set of functions $f_{1}(z), \ldots, f_{m}(z)$ 
analytic in some domain which constitutes a solution of the system of linear homogeneous equations (13) if it is linearly independent over $\mathbf{C}(z)$ and an equation

$$
\sum_{k=1}^{m} P_{k}(z) y_{k}=0, \quad P_{k}(z) \in \mathbf{C}[z], \quad k=1, \ldots, m,
$$

where $\mathrm{y}_{1}, \ldots, y_{m}$ is some solution of the system (13), can take place only in the case when $P_{k}(z) y_{k}=0, k=1, \ldots, m$, is identically in $z$.

Let $l_{\mathbf{K}}=l_{\mathbf{K}}\left(z_{1}, \ldots, z_{m}\right)$ be an arbitrary linear form, not identically zero, with coefficients from $Z_{\mathbf{K}}$ of height $H$ with respect to $\mathrm{K}$ and $l_{\mathbf{K}_{\mathbf{i}}}=l_{\mathbf{K}_{i}}\left(z_{1}, \ldots, z_{m}\right), i=1, \ldots, n$, be linear forms obtained from the form $l_{\mathbf{K}}$ by replacing all its coefficients with their conjugates from $\mathbf{K}_{i}$.

\section{The estimates of the measures of linear independence}

THEOREM 1. Let the set of KE-functions (7), $m \geqslant 2$, constitute a solution of the system of linear homogeneous differential equations (13) and be linearly independent over $\mathbf{C}(z), \xi \in \mathbf{K}$ and $\xi T^{\circ}(\xi) \neq 0$. Then there exist constants $\gamma$ and $\Omega(m)$, such that if $\ln \ln H>\Omega(m)$ the inequality

$$
\max _{1 \leqslant i \leqslant h}\left|l_{\mathbf{K}_{i}}\left(f_{1, i}\left(\xi_{i}\right), \ldots, f_{m, i}\left(\xi_{i}\right)\right)\right|>H^{\rho}
$$

holds and if $k=\mathbf{I}$ then the inequality

$$
L_{\mathrm{l}}\left(f_{1}(\xi), \ldots, f_{m}(\xi) ; H\right)>H^{p}
$$

holds where $\rho=1-m-\gamma m^{7 / 2}(\ln \ln H)^{-\frac{1}{2}}$.

COROLLARY. Under the conditions of Theorem 1 there exist constants $y$ and $\Omega_{1}(m)$, such that for any $H$ the inequality

$$
L_{\mathbf{1}}\left(f_{1}(\xi), \ldots, f_{m}(\xi) ; H\right)>\Omega_{1}(m) H^{\rho}
$$

holds where $\rho=1-m-\gamma m^{7 / 2}(\ln \ln (H+2))^{-\frac{1}{2}}$.

THEOREM 1'. If under the conditions of Theorem 1 the functions (7) constitute an irreducible set of functions, then in the statements of Theorem 1 and its corollary,

where

$$
\Omega(m)=\gamma^{2} m^{5}, \quad \Omega_{1}(m)= \begin{cases}1, & \text { if } \rho<1, \\ \exp \left\{-(2 m-1) \exp \left(\gamma^{2} m^{5}\right)\right\}, & \text { if } \rho \geqslant 1,\end{cases}
$$

$$
\rho=\gamma m^{5 / 2}\left(\ln \ln (H+2)^{-\frac{1}{2}} .\right.
$$

Theorem 1' states that under the condition of the irreducibility of the considered set of functions, all the constants involved in the statements of Theorem 1 and its corollary are effective. 
For the proof of the theorems formulated, we establish some auxiliary propositions.

LEMMA 1 (see [5]). Let the coefficients of the linear forms

$$
L_{k}=a_{k, 1} x_{1}+\ldots+a_{k, q} x_{q}, \quad k=1, \ldots, p, \quad p<q,
$$

belong to $\mathbf{Z}_{\mathbf{K}}$ and

$$
a_{k, i}^{*} \leqslant A, \quad A>0, \quad k=1, \ldots, p, \quad i=1, \ldots, q .
$$

Then there exists a nontrivial solution $x_{1}, \ldots, x_{q}, x_{i} \in \mathbf{Z}_{\mathbf{K}}, i=1, \ldots, q$, of the system of equations $L_{\mathrm{k}}=0, k=1, \ldots, p$, such that

$$
x_{i}^{*}<\eta(\eta q A)^{p(q-p)^{-1}}, \quad i=1, \ldots, q,
$$

where $\eta$ is a constant dependent only on the field $\mathbf{K}$.

LEMMA 2. Let $f_{k}(z) \in \mathbf{K} E(\lambda, c ; \mu, q), k=1, \ldots, m, n \in \mathbf{N}$, and $\omega=\omega(n)$ be a nondecreasing function of $n, 2 \leqslant \omega(n) \leqslant n$. Then there exist $m$ polynomials

$$
P_{k}(z)=\sum_{l=0}^{n} b_{k, l} z_{l}, \quad k=1, \ldots, m,
$$

not all identically zero with the following properties

(1) $b_{k, l} \in \mathbf{Z}_{\mathbf{K}}, l=0,1, \ldots, n, k=1, \ldots, m$,

(17) $\quad b_{k, l}^{*}<n^{n} \exp \left(\sigma \ln \left(2 c q_{0}\right) m^{2} \omega n\right), \quad l=0,1, \ldots, n, \quad k=1, \ldots, m ;$

(2) the linear form

$$
R=\sum_{k=1}^{m} P_{k}(z) f_{k}(z)=\sum_{v=0}^{\infty} a_{v} \frac{z^{v}}{v !},
$$

vanishes at $z=0$ to the order not less than $m(n+1)-\left[n \omega^{-1}\right]-1$, so that

$$
a_{v}=0, \quad v=0,1, \ldots, m(n+1)-\left[n \omega^{-1}\right]-2 ;
$$

(3) the coefficients $a_{v}$ of the form (18) satisfy the condition

$$
a_{v}^{*}<n^{n}(2 c)^{v} \exp \left(\sigma_{0} \ln \left(2 c q_{0}\right) m^{2} \omega n\right), \quad v \geqslant m(n+1)-\left[n \omega^{-1}\right]-1 .
$$

Proof. Let

$$
P_{k}(z)=n ! \sum_{v=0}^{n} g_{k, v} \frac{z^{v}}{v !}, \quad k=1, \ldots, m,
$$

where $g_{k, v} \in \mathbf{Z}_{\mathbf{K}}$. Then $P_{k}(z) \in \mathbf{Z}_{\mathbf{K}}[z]$.

Representing the considered $K E$-functions in the form (7), set

$$
P_{k}(z) f_{k}(z)=n ! \sum_{v=0}^{\infty} d_{k, v} \frac{z^{v}}{v !}, \quad k=1, \ldots, m,
$$


where

$$
d_{k, v}=\sum_{\rho=0}^{n}\left(\frac{v}{\rho}\right) g_{k, \rho} c_{k, v-\rho}
$$

as $g_{k, \rho}=0$ while $\rho>n$. Then

$$
a_{v}=n ! \sum_{k=1}^{m} d_{k, v}, \quad v=0,1,2, \ldots
$$

On multiplying both parts of the equalities (24) by $q_{0, v} / n$ !, where $\left\{q_{0, v}\right\}$ is the sequence (8), satisfying conditions (9) according to the conditions (19) and equalities (23) we obtain $m(n+1)-\left[n \omega^{-1}\right]-1$ linear homogeneous equations for finding out $m(n+1)$ unknown coefficients $g_{k, \rho}$ :

$$
\sum_{k=1}^{m} \sum_{\rho=0}^{n} q_{0, v}\left(\frac{v}{\rho}\right) c_{k, v-\rho} g_{k, \rho}=0, \quad v=0,1, \ldots, m(n+1)-\left[n \omega^{-1}\right]-2 .
$$

As in the equations (25), $v \leqslant m(n+1)$, we have because of (11)

$$
\begin{gathered}
q_{0, v} \leqslant \mu_{0} q_{0}^{m(n+1)} \leqslant \mu^{m} q_{0}^{m(n+1)}, \quad\left(\frac{v}{\rho}\right) \leqslant 2^{m(n+1)}, \quad c_{k, v}^{*} \leqslant \lambda c^{m(n+1)}, \\
\left(q_{0, v}\left(\frac{v}{\rho}\right) c_{k, v-\rho}\right)^{*} \leqslant \lambda \mu^{m}\left(2 c q_{0}\right)^{m(n+1)} .
\end{gathered}
$$

Applying Lemma 1 to the system of equations (25) with

$$
p=m(n+1)-\left[n \omega^{-1}\right]-1, \quad q=m(n+1), \quad A=\lambda \mu^{m}\left(2 c q_{0}\right)^{m(n+1)},
$$

because of the inequality

$$
p(q-p)^{-1}=\left(m(n+1)-\left[n \omega^{-1}\right]-1\right)\left(\left[n \omega^{-1}\right]+1\right)^{-1}<m(n+1) n^{-1} \omega \leqslant 2 m \omega,
$$

we obtain $g_{k, \rho} \in \mathbf{Z}_{\mathbf{K}}$ not all equal to zero and according to the inequality (16) such that

$$
\overline{\left|g_{k, \rho}^{+}\right|}<\eta\left\{\eta m n \lambda \mu^{m}\left(2 c q_{0}\right)^{m(n+1)}\right\}<\exp \left(\sigma, \ln \left(2 c q_{0}\right) m^{2} \omega n\right) .
$$

From equalities (21) and inequalities (26), we obtain the estimates (17) and from equalities (23), (24) and inequalities (26) we obtain the estimates (20). The lemma is proved.

Set

$$
t=\left[n \omega^{-1}\right]+p+\frac{1}{2} g m(m-1)
$$

where $n$ and $\omega$ have the same sense as in Lemma 2 and $p$ and $g$ are defined above. 
Let

$$
R_{1}=\sum_{l=1}^{m} P_{1, l} f_{l}(z), \quad P_{1, l} \in \mathbf{Z}_{\mathbf{K}}[z], \quad l=1, \ldots, m,
$$

be an arbitrary linear form of functions (7). Set

$$
R_{k}=T^{0} R_{k-1}^{\prime}, \quad k=2,3, \ldots,
$$

where $T^{0} R_{k-1}^{\prime}$ is a linear form which arises after differentiation of the form $R_{k-1}$ by $z$ and substitution of the right-hand side of the differential equations of system (13) for $f_{1}^{\prime}(z), \ldots, f_{m}^{\prime}(z)$ and multiplying them by $T^{0}$. Then

$$
R_{k}=\sum_{l=1}^{m} P_{k, l} f_{l}(z), \quad k=1,2, \ldots, \quad P_{k, l} \in \mathbf{Z}_{\mathbf{K}}[z]
$$

Let us denote by

$$
\Delta=\Delta(z)=\left|P_{k, l}\right|_{k, l=1, \ldots, m},
$$

the determinant of the set of linear forms $R_{1}, \ldots, R_{m}$.

LEMMA 3. Let the functions $f_{k}(z) \in \mathbf{K} E(\lambda, c ; \mu, q), k=1, \ldots, m$, constitute a solution of the system of linear homogeneous differential equations (13) and be linearly independent over $\mathbf{C}(z)$. Let further the linear approximating form $R_{1}(28)$ be constructed by Lemma 2 with some $n$. Then there exists a number $n_{0} \in \mathbf{N}$ such that for any $n \geqslant n_{0}$ the determinant $\Delta$ (30) of the system of linear forms $R_{1}, \ldots, R_{m}(29)$ does not vanish identically and is of the form

$$
\Delta(z)=z^{m(n+1)-[n \omega-1]-m-p} \Delta_{1}(z), \quad \Delta_{1}(z) \neq 0, \quad n \geqslant n_{0},
$$

where $\Delta_{1}(z) \in \mathbf{Z}_{\mathbf{k}}[z]$ and is of degree $r_{1}, 0 \leqslant r_{1} \leqslant t$.

The proof of Lemma 3 is quite analogous to the proof of Lemma 6 in [7] with the use of Lemma 2 and with some formal changes which arise because of Lemma 2. Then it occurs that

$$
n_{0}=\delta+\frac{1}{2} g m(m-1)
$$

and if the considered functions constitute an irreducible set of functions then by Lemma 4 from [11]

$$
n_{0}=p+\frac{1}{2} g m(m-1)
$$

LEMMA 4. Let the functions $f_{k}(z), k=1, \ldots, m$, satisfy all the conditions of Lemma 3, the number $n_{0}$ be defined by (32) (and in the corresponding case by (33)) and the number $t$ by (27), $\alpha \in \mathrm{C}, \alpha T^{\circ}(\alpha) \neq 0$, and the linear form $R_{1}=R_{1}(z)(26)$ be constructed 
by Lemma 3 for any $n \geqslant n_{0}$. Then the matrix of coefficients of the linear forms $R_{1}(\alpha), \ldots, R_{m+\tau}(\alpha)((29))$

$$
\left\|P_{k, l}(\alpha)\right\|, \quad k=1, \ldots, m+t, \quad l=1, \ldots, m,
$$

has rank $m$.

Because of Lemma 3, Lemma 4 may be proved quite similarly to Lemma 7 from [7].

LEMMA 5. Let the functions $f_{k}(z) \in \mathbf{K} E(\lambda, c ; \mu, q), k=1, \ldots, m$, constitute a solution of the system of the linear homogeneous differential equations (13) and $\xi \in \mathbf{K}$. Further let the linear form $R_{1}=R_{1}(z)(28)$ be constructed by Lemma 2 for some $n$ and the number $t$ be defined by (27). Then for any $k$, satisfying the inequality $k \leqslant m+t$ the estimates

$$
\begin{aligned}
\overline{\left|P_{k, l}(\xi)^{k}\right|} & <n^{n} \exp \left(\gamma_{1} m n\left(\ln \left(2 c q_{0}\right) \ln n\right)^{\frac{1}{2}}\right) \\
\left|R_{k}(\xi)\right| & <n^{-(m-1) n} \exp \left(\gamma_{2} m n\left(\ln \left(2 c q_{0}\right) \ln n\right)^{\frac{1}{2}}\right),
\end{aligned}
$$

hold and the estimate (36) remains valid if we change all the coefficients of the power series $R_{k}(z)$ and number $\xi$ to their conjugates from any field $\mathbf{K}_{i}$.

PRoor. If the coefficients of the power series

satisfy the conditions

$$
\Phi(z)=\sum_{k=0}^{\infty} \alpha_{k} z^{k}, \quad \Psi(z)=\sum_{k=0}^{\infty} \beta_{k} z_{k},
$$

$$
\left|\alpha_{k}\right| \leqslant \beta_{k}, \quad \beta_{k} \geqslant 0, \quad k=1,2, \ldots,
$$

then we shall write $\Phi \ll \Psi$. Besides that we shall denote

First of all we have

$$
\Phi(z)=\sum_{k=0}^{\infty}\left|\alpha_{k}\right| z^{k}, \quad \Phi(z) \ll \hat{\Phi}(z) .
$$

$$
T^{0}(z) \ll G(1+z)^{g}, \quad T^{0}(z) Q_{k, i}(z) \ll G(1+z)^{q},
$$

where the numbers $g$ and $G$ are defined above.

Repeating word for word the arguments of Lemma 6 from [5] we obtain by induction that

$$
\begin{gathered}
P_{k+1, l}(z) \ll G^{k}(1+z)^{k g+n} \exp \left(\ln \left(2 c q_{0}\right) m^{2} \omega n\right) n^{n} \prod_{v=0}^{k-1}(v g+m+n), \\
R_{k+1}(z) \ll G^{k}(1+z)^{k g} \prod_{v=0}^{k-1}\left(v g+\frac{d}{d z}\right) \hat{R}_{1}(z), \quad k=0,1,2, \ldots,
\end{gathered}
$$

and the relations (38) and (39) remain valid if we change the coefficients of the polynomial $P_{k+1, l}(z)$ and of the power series $R_{k}(z)$ by their conjugates from any field $\mathbf{K}_{\boldsymbol{i}}$. 
We have $n \geqslant n_{0}=\delta+\frac{1}{2} g m(m-1)$. Suppose that $n$ and $\omega$ are such that

$$
n \omega^{-1}>2 m^{2} \ln 2 \text {. }
$$

Then

$$
k \leqslant m+t=m+\left[n \omega^{-1}\right]+p+\frac{1}{2} g m(m-1)<\gamma_{3} n \omega^{-1} .
$$

Now from (38) and (41) it follows that

$$
P_{k, l}^{*}(\xi)<\exp \left(\sigma \ln \left(2 c q_{0}\right) m^{2} \omega n+\gamma_{4} n \omega^{-1} \ln n\right) n^{n}, \quad k \leqslant m+t .
$$

Further from (41) and (20) we have, setting $a=m(n+1)-\left[n \omega^{-1}\right]-1$,

$$
\begin{aligned}
& \prod_{v=0}^{k-1}\left(v g+\frac{d}{d z}\right) \hat{R}_{1}(z) \ll \exp \left(\gamma_{5} n \omega^{-1} \ln n\right)\left(1+\frac{d}{d z}\right)^{k} \hat{R}_{1}(z) \\
& \ll \exp \left(\sigma_{0} \ln \left(2 c q_{0}\right) m^{2} \omega n+\gamma_{5} n \omega^{-1} \ln n\right) n^{n}\left(1+\frac{d}{d z}\right)^{k} \sum_{v=a}^{\infty}(2 c)^{\nu} \frac{z^{v}}{v !} \\
& \ll \exp \left(\sigma_{0} \ln \left(2 c q_{0}\right) m^{2} \omega n+\gamma_{5} n \omega^{-1} \ln n\right) n^{n} \sum_{\rho=0}^{k} \sum_{\nu=a}\left(\frac{k}{\sigma}\right)(2 c)^{v} \frac{z^{v-\rho}}{(v-\sigma) !} \\
& \ll \exp \left(\sigma_{0} \ln \left(2 c q_{0}\right) m^{2} \omega n+\gamma_{5} n \omega^{-1} \ln n\right) n^{n} 2^{k} \sum_{\nu=a-k}(2 c)^{v+k} \frac{z^{v}}{v !}
\end{aligned}
$$

Thus we obtain

(43) $\left|\left\{\prod_{v=0}^{k-1}\left(v q+\frac{d}{d z}\right) \hat{R}_{1}(z)\right\}\right|<\exp \left(\sigma_{0} \ln \left(2 c q_{0}\right) m^{2} \omega n+\gamma_{6} n \omega^{-1} \ln n\right) n^{-(m-1) n}$.

From (39) and (43) we find that

$$
\left|R_{k}(\xi)\right|<\exp \left(\sigma_{0} \ln \left(2 c q_{0}\right) m^{2} \omega n+\gamma_{7} n \omega^{-1} \ln n\right) n^{-(m-1) n}, \quad k \leqslant m+t .
$$

Making equal the parts of the exponent in the right-hand side of the inequality (42) we set

$$
\omega=m^{-1}(\ln n)^{\frac{1}{2}}\left(\ln \left(2 c q_{0}\right)\right)^{-\frac{1}{2}} .
$$

As $\omega \geqslant 2$ by given data, $n$ must satisfy the condition $\ln n \geqslant 4 m^{2} \ln \left(2 c q_{0}\right)$. Then

$$
n \omega^{-1}=m n\left(\ln \left(2 c q_{0}\right)\right)^{\frac{1}{2}}(\ln n)^{-\frac{1}{2}}>(\ln 2)^{\frac{1}{2}} m n^{\frac{1}{2}}>2 m^{2} \ln 2,
$$

because $c q_{0}>1$ and $n^{\frac{1}{2}}>(\ln n)^{\frac{1}{2}}>2 m(\ln 2)^{\frac{1}{2}}$. That means that (40) is true.

Putting the value $\omega(45)$ into the estimates (42) and (44) we obtain the assertions (35) and (36) of the lemma. 
ProOf OF TheOReM. 1. Let us consider an arbitrary linear form

$$
L_{0}=u_{1} f_{1}(\xi)+\ldots+u_{m} f_{m}(\xi), \quad\left|u_{1}\right|+\ldots+\left|u_{m}\right|>0, \quad u_{l} \in \mathbf{Z}_{\mathbf{k}}, \quad u_{l}^{*} \leqslant H, \quad l=1, \ldots, m .
$$

Let the functional linear form $R_{1}(z)$ (28) be constructed by Lemma 2 with some $n$ and the forms $R_{k}(z), k=1,2 \ldots$, are determined by the form $R_{1}(z)$ in (29).

As the $K E$-functions (7) are linearly independent over $\mathbf{C}(z)$ and $\xi T^{\circ}(\xi) \neq 0$ then by Lemmas 3, 4 and 5 for any $n \geqslant n_{0}$ where $n_{0}$ is defined in (32) among the numerical linear forms

$$
R_{k}(\xi), \quad k=1, \ldots, m+t, \quad m+t<\gamma_{3} n \omega^{-1},
$$

we can select $m$ linearly independent while estimates (35) and (36) hold and

$$
\max \operatorname{deg} P_{k, l}(z) \leqslant \gamma_{8} n, \quad k=1, \ldots, m+t, \quad l=1, \ldots, m .
$$

Then among the linear forms (46) we can select $m-1$ forms such that together with $L_{0}$ they are linearly independent. Let these forms be

$$
L_{s}=R_{k_{s}}(\xi), \quad k_{s} \leqslant m+t, \quad s=1, \ldots, m-1 .
$$

Denote by $\Delta, \Delta \neq 0$, the determinant of the linear forms (48) and $L_{0}$ and by $\Delta_{k, l}$ the cofactor of the element of $\Delta$ in the $k$ th row and the $l$ th column. Then we have

$$
f_{l}(\xi) \Delta=\Delta_{1, l} L_{1}+\ldots+\Delta_{m-1, l} L_{m-1}+\Delta_{m, l} L_{0}, \quad 1 \leqslant l \leqslant m .
$$

Let us take $l$ such that $f_{l}(\xi) \neq 0$, which is quite possible because $T^{0}(\xi) \neq 0$. Then it follows from (49) that

$$
\left|\Delta_{m, l}\right|\left|L_{0}\right| \geqslant\left|f_{l}(\xi)\right||\Delta|-(m-1) \max _{1 \leqslant k \leqslant m-1}\left|\Delta_{k, l}\right| \max _{1 \leqslant k \leqslant m-1}\left|L_{k}\right| .
$$

With the help of the estimates (35) and (36) we obtain the inequalities

$$
\begin{aligned}
\mid \overline{\Delta_{m, l}^{k} \mid} & <\exp \left(\gamma_{9} m^{2} n\left(\ln \left(2 c q_{0}\right) \ln n\right)^{\frac{1}{t}}\right) n^{(m-1) n}, \\
\max _{1 \leqslant k \leqslant m-1} \overline{\left|\Delta_{k, l}^{k}\right|} & <\exp \left(\gamma_{10} m^{2} n\left(\ln \left(2 c q_{0}\right) \ln n\right)^{\frac{1}{1}}\right) n^{(m-2) n}, \\
\max _{1 \leqslant k \leqslant m-1}\left|L_{k}\right| & <\exp \left(\gamma_{2} m^{2} n\left(\ln \left(2 c q_{0}\right) \ln n\right)^{\frac{1}{2}}\right) n^{-(m-1) n} .
\end{aligned}
$$

Let $a \in \mathbf{N}$ be such that $a \xi \in \mathbf{Z}_{\mathbf{K}}$. Then it follows from (47) that $a^{\gamma^{n}} \Delta=e^{\gamma_{11^{n}}} \Delta \in \mathbf{Z}_{\mathbf{K}}$, and this implies, because $\Delta \neq 0$, that

$$
\left(e^{\gamma_{11}}\left|\Delta_{1}\right|\right) \ldots\left(e^{\gamma_{11}}\left|\Delta_{h}\right|\right) \geqslant 1,
$$

where $\Delta_{1}, \ldots, \Delta_{h}$ are the conjugates to $\Delta$. Thus, for some $i$,

$$
\left|\Delta_{i}\right|>e^{-\gamma_{11} n} \text {. }
$$


Let us replace the field $\mathbf{K}$ by the field $\mathbf{K}_{i}$. That means, that in the power series of all the functions (7) in the form $L_{0}$ all the coefficients are replaced by their conjugates from the field $\mathbf{K}_{i}$ and the number $\xi$ by $\xi_{i}$.

It is evident that after such a replacement all the arguments and estimates given above remain valid. The inequality (50) will take the form

$$
\Delta_{m, l}^{*}\left|L_{0, i}\right| \geqslant\left|f_{l, i}\left(\xi_{i}\right)\right|\left|\Delta_{i}\right|-(m-1) \max _{1 \leqslant k \leqslant m-1} \Delta_{k, l}^{*} \max _{1 \leqslant k \leqslant m-1}\left|L_{k, i}\right|,
$$

where $L_{k, i}$ denotes the linear form, corresponding in the field $\mathbf{K}_{i}$ to the form $L_{k}$. If we replace the left-hand side of (52) by $\left|L_{k, i}\right|$ then, by Lemma 5 , this inequality remains valid.

From inequalities (54), (51), (52) and (53) we deduce that

$$
\begin{aligned}
&\left|L_{0, i}\right|>\exp \left(-\gamma_{12} m^{2} n\left(\ln \left(2 c q_{0}\right) \ln n\right)^{\frac{1}{2}}\right) n^{-(m-1) n} \\
& \times\left(1-H n^{-n} \exp \left(\gamma_{13} m^{2} n\left(\ln \left(2 c q_{0}\right) \ln n\right)^{\frac{1}{2}}\right)\right) .
\end{aligned}
$$

Take the minimal $n$ such that the conditions

$$
n \geqslant n_{0}, \quad(\ln n)^{\frac{1}{2}}>2 \gamma_{13}\left(\ln \left(2 c q_{0}\right)^{\frac{1}{2}} m^{2},\right.
$$

and

$$
n^{n} \exp \left(-\gamma_{13} m^{2} n\left(\ln \left(2 c q_{0}\right) \ln n\right)^{\frac{1}{2}}\right)>2 H
$$

are satisfied. Then from inequality (55) it follows that

$$
\left|L_{0, i}\right|>\frac{1}{2} \exp \left(-\gamma_{12} m^{2} n\left(\ln \left(2 c q_{0}\right) \ln n\right)^{\frac{1}{2}}\right) n^{-(m-1) n} .
$$

Under our assumptions beginning with some $H$ the condition

$$
(n-1)^{(n-1)} \exp \left(\gamma_{13} m^{2}(n-1)\left(\ln \left(2 c q_{0}\right) \ln (n-1)\right)^{\frac{1}{1}}\right) \leqslant 2 H,
$$

holds and this implies that

$$
\begin{aligned}
& n^{n} \exp \left(-\gamma_{13} m^{2} n\left(\ln \left(2 c q_{0}\right) \ln n\right)^{\frac{1}{2}}\right) \leqslant 2 n H\left(1+(n-1)^{-1}\right)^{n-1} \\
& \quad \times \exp \left(-\gamma_{13} m^{2}\left(\ln \left(2 c q_{0}\right)\right)^{\frac{1}{2}}\left(n(\ln n)^{\frac{1}{2}}-(n-1)(\ln (n-1))^{\frac{1}{2}}\right)\right)<2 e H n .
\end{aligned}
$$

Taking logarithms, we obtain the inequality

$$
n \ln -\gamma_{13} m^{2} n\left(\ln \left(2 c q_{0}\right) \ln n\right)^{\frac{1}{2}}<\ln (2 e)+\ln H+\ln n,
$$

and because of the inequality (56), that implies that

$$
n \ln n<\gamma_{14} \ln H \text {, }
$$

and then, in accordance with inequalities (57), (59) and (60), we have

$$
2 H<n^{n} \exp \left(-\gamma_{13} m^{2} n\left(\ln n \ln \left(2 c q_{0}\right)\right)^{\frac{1}{2}}\right)<\gamma_{15} H
$$

and

$$
\ln \ln H>\gamma_{16} \ln n \text {. }
$$


From (58), (60) and (61) we deduce that

$$
\left|L_{0, i}\right|>H^{a} \text {, }
$$

where $a=1-m-\gamma_{17} m^{3}\left(\ln \left(2 c q_{0}\right)\right)^{\frac{1}{2}}(\ln \ln H)^{-\frac{1}{2}}$ and as, according to (11) $q_{0}=q^{m}$ and in view of inequalities (56) and (62), we have

$$
\left|L_{0, i}\right|>H^{b}
$$

where $b=1-m-\gamma_{18} m^{7 / 2}(\ln \ln H)^{-\frac{1}{2}}$, while

Set

$$
\ln \ln H>\max \left(\gamma_{19} m^{5}, \gamma_{16} n_{0}\right) \text {. }
$$

$$
\gamma=\max \left(\gamma_{18}, \gamma_{19}^{\frac{1}{1}}\right), \quad \Omega(m)=\max \left(\gamma^{2} \mathrm{~m}^{5}, \gamma_{16} \ln n_{0}\right) .
$$

Then since $\ln \ln H>\Omega(m)$, inequality (14) follows from inequality (63).

If $\mathbf{K}=\mathbf{I}$ then inequality (14) implies inequality (15) because the moduli of the complex conjugate numbers are equal.

The corollary and Theorem $1^{\prime}$ now follow from this theorem and equalities (33) and (64) in view of the fact that since $\ln \ln H \leqslant \gamma^{2} m^{5}$ the inequality

$$
L_{\mathbf{1}}\left(f_{1, i}\left(\xi_{i}\right), \ldots, f_{m, i}\left(\xi_{i}\right) ; H\right) \geqslant L_{\mathbf{1}}\left(f_{1, i}\left(\xi_{i}\right), \ldots, f_{m, i}\left(\xi_{i}\right) ;\left[\exp \exp \left(\gamma^{2} m^{5}\right)-1\right]\right)
$$

holds.

Theorem 1 implies a very useful theorem for applications to concrete functions.

THEOREM 2. Let the set of $K E$-functions (7), $m \geqslant 1$, constitute a solution of the system of linear differential equations (12) and be linearly independent with the number 1 over $\mathbf{C}(z)$ and let $\xi \in \mathbf{K}, \xi T(\xi) \neq 0$. Then there exist constants $\gamma$ and $\Omega(m)$ such that under the condition $\ln \ln H>\Omega(m)$ the inequality

$$
\max _{1 \leqslant i \leqslant h} \mid l_{\mathbf{K}_{i}}\left(1, f_{1, i}\left(\xi_{i}\right), \ldots, f_{m, i}\left(\xi_{i}\right) \mid>H^{a}\right.
$$

is true and if $\mathbf{K}=\mathbf{I}$ then the inequality

$$
L_{1}\left(f_{1}, f_{1}(\xi), \ldots, f_{m}(\xi) ; H\right)>H^{a}
$$

is true where $a=-m-\gamma m^{7 / 2}(\ln \ln H)^{-\frac{1}{2}}$.

The corollary to Theorem 2 and Theorem $2^{\prime}$ analogous to the corollary to Theorem 1 and Theorem 1 ' holds.

\section{The estimates of the algebraic independence measures}

The proof of Theorem 1 and Lemmas 6, 7 and 8 given below implies some more general assertions. 
THEOREM 3. Let the set of $\mathbf{I}$-functions (7), $m \geqslant 1$, constitute a solution of the system of the linear differential equations (12) and be algebraically independent over $\mathbf{C}(z)$, and $\xi \in \mathbf{I}, \xi T(\xi) \neq 0$. Then there exist constants $\gamma$ and $\Omega(m)$ such that the inequality

holds, where

$$
\varphi_{\mathbf{I}}\left(f_{1}(\xi), \ldots, f_{m}(\xi) ; n ; H\right)>C H^{a}
$$

$$
a=1-(n+m) !(n ! m !)^{-1}-\gamma(n+1)^{3 m}(m \ln (n+1))^{\frac{1}{2}}(\ln \ln (H+2))^{-\frac{1}{2}},
$$

and

$$
C= \begin{cases}1, & \text { if } \rho<1, \\ \exp \left\{-\left((n+1)^{m}\left(1+\frac{\gamma}{\Omega(m)}\right)-1\right) \exp \left(\Omega^{2}(m) m(n+1)^{4 m} \ln (n+1)\right)\right\}, & \text { if } \rho \geqslant 1,\end{cases}
$$

where

$$
\rho=\Omega(m)(n+1)^{2 m}(m \ln (n+1))^{\frac{1}{2}}(\ln \ln (H+2))^{-\frac{1}{2}} .
$$

THEOREM 3'. If under the conditions of Theorem 3 the set of products

$$
f_{1}^{k_{1}}(z) \ldots f_{m}^{k_{m}}(z), \quad 0 \leqslant k_{1}+\ldots+K_{m} \leqslant N,
$$

constitutes for any $N=1,2, \ldots$, an irreducible set of functions then $\Omega(m)=\gamma$.

Lemma 6. Let the functions (7) belong to $\mathrm{K} E(\lambda, c ; \mu, q)$ and let $N \in \mathbf{N}$. Then $M=(N+m) ! / N ! m !$, products (66) belong to the class

$$
\mathbf{K} E\left(\lambda^{N}, N c ; \mu^{m N}, q^{m(\ln N+1)}\right),
$$

and the sequence $\left\{q_{n}\right\}$ involved in the definition of class (67) may be selected suitable for all the $M$ functions (66), that is,

$$
\left.\mu_{0}=\mu^{m N}, \quad q_{0}=q^{m(\ln N+1}\right) .
$$

Proof. Consider one of the products (66) for which $k_{1}+\ldots+k_{m}=s, 1 \leqslant s \leqslant N$. The product (66) may be considered as the product of some $s$ of the functions (7); some factors may be repeated. Arrange these $s$ functions in an arbitrary order and denote the coefficients of their power series by $\gamma_{l, n} / n !, n=0,1, \ldots, l=1, \ldots, s$, correspondingly. Then

$$
f_{1}^{k_{1}}(z) \ldots f_{m}^{k_{m}}(z)=\sum_{n=0}^{\infty} \frac{\gamma_{n}}{n !} z^{n}
$$

where

$$
\gamma_{n}=\sum_{n_{1}+\ldots+n_{s}=n} \frac{n !}{n_{1} ! \ldots n_{s} !} \gamma_{1, n_{1}} \ldots \gamma_{s, n_{s}}
$$


According to the definition of the class $\operatorname{KE}(\lambda, c ; \mu, q)$ we have

$$
\begin{aligned}
\gamma_{n}^{*} & \leqslant \sum_{n_{1}+\ldots+n_{s}=n} \frac{n !}{n_{1} ! \ldots n_{s} !} \gamma_{1, n_{1}}^{*} \ldots \gamma_{s, n_{s}}^{*} \\
& \leqslant \lambda^{s} c^{n} \sum_{n_{1}+\ldots+n_{s}=n} \frac{n !}{n_{1} ! \ldots n_{s} !} \lambda^{s} c^{s} s^{n} \leqslant \lambda^{N}(c N)^{n} .
\end{aligned}
$$

For any $n$ in any item, involved in the right-hand side of (69), we arrange the factors $c_{l, n}$ so that the second index does not increase, that is,

$$
\gamma_{1, n_{1}} \ldots \gamma_{s, n_{s}}=\gamma_{l_{1}, n_{l_{1}}} \ldots \gamma_{l_{s}, n_{l_{s}}}, \quad n_{l_{1}} \geqslant n_{l_{2}} \geqslant \ldots \geqslant n_{l_{s}} .
$$

Then it is evident that $n_{l_{1}} \leqslant n, n_{l_{2}} \leqslant n / 2, \ldots, n_{l_{s}} \leqslant n / s$. Thus if we set

$$
q_{n}=q_{0, n} q_{0,[n / 2]} \ldots q_{0,[n / N]}
$$

where $\left\{q_{0, n}\right\}$ is the sequence $(10)$ then $q_{n} \gamma_{l} \in \mathbf{Z}_{\mathbf{K}}, l=0,1, \ldots, n$ and $n=1,2, \ldots$, and in view of (8) and (11) we obtain

$$
q_{n} \leqslant \mu^{m s} q^{m\left(1+\frac{1}{2}+\ldots+1 / N\right) n} \leqslant \mu^{m N} q^{m \ln (N+1) n} .
$$

The lemma is proved.

Let $\Phi(z)$ be a function, analytical at $z=0$. Denote by $O(\Phi(z))$ the order of zero of $\Phi(z)$ at $z=0$.

Lemma 7 (Nesterenko, see [10], Theorem 3). Let $P \in \mathbf{C}\left[z, x_{1}, \ldots, x_{m}\right], P \not \equiv 0$, $\operatorname{deg}_{z} P \leqslant n, \operatorname{deg}_{\bar{x}} \leqslant N$. Suppose that the functions $f_{1}(z), \ldots, f_{m}(z)$ analytical at $z=0$ constitute a solution of the system of the linear differential equations (12) and are algebraically independent over $\mathbf{C}(z)$. Set $R(z)=P\left(z, f_{1}(z) \ldots, f_{m}(z)\right)$. Let the dimension of the vector space over $\mathbf{C}(z)$ generated by the functions $R^{(i)}(z), i=0,1, \ldots$, be equal to 1 . Then

$$
O(R(z)) \leqslant \ln +\Omega(m) N^{\tau}, \quad \tau=(m+1)^{m+1}+m+1 .
$$

Let the $K E$-functions (7) satisfy the system of the linear differential equations (12). Consider the set of $M=(N+m) ! / N ! m$ ! products (66). By Lemma 12 from [7] the functions (66) constitute a solution of a system of linear homogeneous differential equations of the form (13) in which the number $m$ is replaced by the number $M$ and the coefficients of this system have no poles, different from the poles of the coefficients of system (12). Numerate the $M$ functions (66) in an arbitrary order and denote them by

$$
\Phi_{1}(z), \ldots, \Phi_{M}(z) \text {. }
$$

Using Lemma 6, it is easy to verify that for the functions (70) the assertion of Lemma 2 , in which $m$ is replaced by $M$, remains valid. If we suppose that the 
functions (7) are algebraically independent over $\mathbf{C}(z)$ then the assertions of Lemmas 3,4 and 5 , in which $m$ is also replaced by $M$, are true for the functions (70). In view of Lemma 7 we can refine now Lemma 3.

LEMMA 8. If in Lemma 3 we replace number $m$ by $M$ and the KE-functions (7) by KE-functions (70) while the functions (7) are algebraically independent over $\mathrm{C}(z)$ then

$$
n_{0}=\Omega_{1}(m) N^{\tau}, \quad \tau=(m+1)^{m+1}+m+1 .
$$

PRoof. Let $R_{1}(z)$ be the linear form of the functions (70), constructed by Lemma 2. By this lemma $O\left(R_{1}(z)\right) \geqslant M n-\left[n \omega^{-1}\right]-1$. But then in view of Lemma 7 we have

$$
M n-\left[n \omega^{-1}\right]-1 \leqslant \ln +\Omega(m) N^{\tau}, \quad \tau=(m+1)^{m+1}+m+1 .
$$

The inequalities (71) imply that $\left(M-l-\omega^{-1}\right) n<\Omega_{1}(m) N^{\tau}+2$. If we assume that $l<M$, then $M-l \geqslant 1$ and by the conditions of Lemma 2 we have $\omega^{-1} \leqslant \frac{1}{2}$. Thus $n<\Omega_{1}(m) N^{\tau}+4$. Then for $n \geqslant 2 \Omega_{1}(m) N^{\tau}+4$ we have $l=M$, which implies the lemma.

In view of the equality

$$
\varphi_{\mathbf{I}}\left(f_{1, i}\left(\xi_{i}\right), \ldots, f_{m, i}\left(\xi_{i}\right) ; N ; H\right)=L_{\mathbf{I}}\left(\Phi_{1, i}\left(\xi_{i}\right), \ldots, \Phi_{M, i}\left(\xi_{i}\right) ; M H\right)
$$

the proof of Theorem 3 is quite analogous to the proof of Theorem 1 with the use of Lemmas 6 and 8.

From inequality (63) and the equalities obtained with the help of Lemma 6 and analogous to equalities (64), we deduce that for

$$
\begin{aligned}
\ln \ln (H+2)>\max & \left(\gamma^{2} m M^{4} \ln (N+1), \gamma_{16} \ln n_{0}\right) \\
& =\max \left(\gamma^{2} m M^{4} \ln (N+1), \gamma_{16}\left(\tau \ln (N+1)+\ln \Omega_{1}(m)\right)\right),
\end{aligned}
$$

and this means that for

$$
\ln \ln (H+2)>\Omega^{2}(m) m(N+1)^{4 m} \ln (N+1)
$$

after replacing number $N$ by $n$ the assertion of Theorem 3 with $C=1$ is true. In the case

$$
\ln \ln (H+2) \leqslant \Omega^{2}(m) m(N+1)^{4 m} \ln (N+1) .
$$

Theorem 3 is proved with the help of the inequality for the functions (70) analogous to inequality (65).

Theorem $3^{\prime}$ follows from the proof of Theorem 3 in view of (33) in which $m$ is replaced by $M$. 
THEOREM 4. Let the set of IE-functions (7), $m \geqslant 2$, constitute a solution of the system of linear homogeneous equations (13) and be not connected by any homogeneous algebraic equation with coefficients from $\mathbf{C}(z)$ and let $\xi \in \mathbf{I}$ and $\xi T^{0}(\xi) \neq 0$. Then there exists constants $\gamma, \gamma_{1}, \Omega(m)$ such that the inequalties

hold where

$$
\begin{array}{r}
\varphi_{\mathrm{I}}^{0}\left(f_{1}(\xi), \ldots, f_{m}(\xi) ; n ; H\right)>C H^{1-M-\delta_{1}}, \\
\varphi_{\mathrm{I}}\left(\frac{f_{1}(\xi)}{f_{m}(\xi)}, \ldots, \frac{f_{m-1}(\xi)}{f_{m}(\xi)} ; n ; H\right)>C H^{1-M-\delta_{1}}
\end{array}
$$

$$
\begin{aligned}
M & =(n+m-1) !(n !(m-1) !)^{-1}, \\
\delta & =\gamma(n+1)^{3(m-1)}(m \ln (n+1))^{\frac{1}{2}}\left(\ln \ln (H+2)^{-\frac{1}{2}},\right.
\end{aligned}
$$

$\delta_{1}$ is the result of replacing $\delta$ by $y$ in $\gamma_{1}$ and

$$
C= \begin{cases}1, & \text { if } \rho<1, \\ \exp \left\{-\left((n+1)^{m-1}\left(1+\gamma \Omega^{-1}(m)\right)-1\right)\right. & \\ \left.\times \exp \left(\Omega^{2}(m) m(n+1)^{4(m-1)} \ln (n+1)\right)\right\}, & \text { if } \rho \geqslant 1,\end{cases}
$$

where

$$
\rho=\Omega(m)(n+1)^{2(m-1)}(m \ln (n+1))^{\frac{1}{2}}(\ln \ln (H+2))^{-\frac{1}{2}} .
$$

The proof of Theorem 4 is analogous to the proof of Theorem 3; we need only to consider $(N+m-1) ! / N !(m-1)$ ! products $(66)$ which satisfy the condition $k_{1}+\ldots+k_{m}=N$.

THEOREM 4'. If in Theorem 4 the set of the products (66) for which $k_{1}+\ldots+k_{m}=N$ constitutes for any $N=1,2, \ldots$ an irreducible set of functions, then $\Omega(m)=\gamma$ and, correspondingly, $\Omega(m)=\gamma_{1}$.

THEOREM 5. Let $s+t>0$ and suppose that any of $s, s \geqslant 0$, sets of the IE-functions

$$
f_{1, l}(z), \ldots, f_{m_{1}, l}(z), \quad m_{l} \geqslant 1, \quad l=1, \ldots, s,
$$

constitute a solution of the corresponding system of linear differential equations

$$
y_{k, l}^{\prime}=Q_{k, 0, l}+\sum_{i=1}^{m_{l}} Q_{k, i, l} y_{i, l}, \quad Q_{k, i, l} \in \mathbf{C}(z), \quad k=1, \ldots, m_{l}, \quad l=1, \ldots, s,
$$

and any of $t, t \geqslant 0$, sets of $\mathbf{I} E$-functions

$$
\Phi_{1, l}(z), \ldots, \Phi_{m_{l}, l}(z), \quad \mu_{l} \geqslant 1, \quad l=1, \ldots, t,
$$

be a solution of the corresponding system of linear homogeneous differential equations

$$
x_{k, l}^{\prime}=\sum_{i=1}^{\mu_{1}} q_{k, i, l} x_{i, l}, \quad q_{k, l, l} \in \mathbf{C}(z), \quad k=1, \ldots, \mu_{l}, \quad l=1, \ldots, t .
$$


Further let $m=m_{1}+\ldots+m_{s}+\mu_{1}+\ldots+\mu_{t}$ functions (72) and (73) be not connected by any algebraic equation with coefficients from $\mathrm{C}[z]$ and homogeneous by any of the $t$ sets of functions (72) and let $\xi \in \mathbf{I}, \xi T_{1}(\xi) \ldots T_{s}(\xi) T_{1}^{0}(\xi) \ldots T_{t}^{0}(\xi) \neq 0$. Then there exist constants $\gamma$ and $\Omega(m)$ such that the inequality

$$
\begin{array}{r}
\varphi_{1}\left(f_{1,1}(\xi), \ldots, f_{m_{1}, 1}(\xi) ; \ldots ; f_{1, s}(\xi), \ldots, f_{m_{s}, s}(\xi) ; \frac{\Phi_{1,1}(\xi)}{\Phi_{\mu_{1}, 1}(\xi)}, \ldots, \frac{\Phi_{\mu_{1}-1,1}(\xi)}{\Phi_{\mu_{1}, 1}(\xi)} ; \ldots ;\right. \\
\left.\frac{\Phi_{1, t}(\xi)}{\Phi_{\mu_{t}, t}(\xi)}, \ldots, \frac{\Phi_{\mu_{t}-1, t}(\xi)}{\Phi_{\mu_{t}, t}(\xi)} ; n_{1}, \ldots, n_{s} ; v_{1}, \ldots, v_{t} ; H\right)>C H^{1-m-\delta},
\end{array}
$$

is true where

$$
\begin{aligned}
& M=\left(\prod_{l=1}^{s} \frac{\left(n_{s}+m_{l}\right) !}{n_{l} ! m_{l} !}\right) \prod_{k=1}^{t} \frac{\left(v_{k}+\mu_{k}-1\right) !}{v_{k} !\left(\mu_{k}-1\right) !}, \\
& \delta=\gamma\left(\prod_{l=1}^{s}\left(n_{l}+1\right)^{3 m_{l}}\right)\left(\prod_{k=1}^{t}\left(v_{k}+1\right)^{3\left(\mu_{k}-1\right)}\right) \\
& \times\left(m \ln \left(n_{1}+\ldots+n_{s}+v_{1}+\ldots+v_{t}+1\right)\right)^{\frac{1}{2}}(\ln \ln (H+2))^{-\frac{1}{2}}
\end{aligned}
$$

and

$$
\rho=B(\ln \ln (H+2))^{-\frac{1}{2}}
$$

The proof of Theorem 5 is analogous to the proofs of Theorems 3 and 4 if we consider $M$ products

(74)

$$
f_{1,1}^{k_{1,1}} \ldots f_{m_{1}, 1}^{k_{m_{1}}, 1} \ldots f_{1, s}^{k_{1, s}} \ldots f_{m_{s}, s}^{k_{m_{1}, s}} \Phi_{1,1}^{\kappa_{1,1}} \ldots \Phi_{\mu_{1}, 1}^{\kappa \mu_{1}, 1} \ldots \Phi_{\mu_{t, i}}^{\kappa \mu_{t, t}}
$$

$$
0 \leqslant k_{1, l}+\ldots+k_{\mu_{l, l}} \leqslant n_{l}, \quad l=1, \ldots, s ; \kappa_{1, k}+\ldots+\kappa_{\mu_{k, k}}=v_{k}, \quad k=1, \ldots, m .
$$

THEOREM 5'. If in Theorem 5 the set of products (74) for any $n_{l}=1,2, \ldots$, $l=1, \ldots, s, v_{k}=1,2, \ldots, k=1, \ldots, t$, constitutes an irreducible set of functions, then $\Omega(m)=\gamma$. 
Note that in Theorems $1-5$ we may consider as the field $\mathbf{K}$ any algebraic number field of a finite degree over $\mathbf{Q}$, which contains all the coefficients of the power series of the considered $E$-functions and the number $\xi$.

The particular cases of Theorem 5 when $s>0, t=0$, and $s=0, t>0$ are of interest. It is easy to reformulate Theorems $1-5$ in the case when the considered functions satisfy linear differential equations but not systems of such equations. For example, we may set, in Theorems 2 and $3, f_{k}(z)=f^{(k-1)}(z), k=1, \ldots, m$, where $f(z)$ is a solution of the equation

$$
P_{m} y^{(m)}+\ldots+P_{1} y^{\prime}+P_{0} y=Q, \quad P_{k}, Q \in \mathrm{C}[z],
$$

and do the same thing with $Q \equiv 0$ in Theorems 1 and 4 . In the case of Theorem 5 we may consider the solutions of several similar equations.

As $\mathbf{Z} \subset \mathbf{K}$, we may omit the indices $\mathbf{K}_{i}$ and $\mathbf{I}$ of the measures $L$ and $\varphi$ in Theorems $1-5$, that is the corresponding assertions are valid for the usual linear independence and algebraic independence measures. With this, in the case of the field $K_{i}$, the index $i$ remains with the $K E$-functions and the number $\xi$.

In view of the fact in the applications of the general theorems to concrete functions, the number $m$ of the considered functions may be arbitrary everywhere in the formulations of the theorems, the dependence of the estimates on $m$ is taken into account.

In all the Theorems $1-5$ the constant $\gamma$ is effective and the constants $\Omega(m)$ and $\Omega_{1}(m)$ are non-effective. The result of $Y u$. V. Nesterenko enabled us to make the constants involved in the estimates effective relative to the degrees of the considered functions $\varphi$, but it does not give the total effect. If we do not use Lemma 7 then all the assertions of Theorems 3-5 remain true but the constants $\mathbf{C}$ will be ineffective relative to all parameters on which they depend.

In Theorems 1'-5' all the constants involved are effective. But we must note that the proofs of the irreducibility of sets of functions are, as usual, very complicated and may be obtained only when these functions are solutions of differential equations of not too high orders.

The estimates of the measures in the theorems proved take place for any values of $m$-the number of the considered functions. But for large enough values of $m$ and the degrees these estimates become rough. But the number $m$ and the degrees of the measures may grow together with $H$ up to some limit and precise enough estimates of the measures remain true.

From the proofs of Theorems 1 and 2 it becomes clear that $m$ may grow depending on the growth of $H$. But the bound of the growth is not indicated effectively. But in Theorems $1^{\prime}$ and $2^{\prime}$ this effective bound is established. For example, in Theorem $1^{\prime}$ for $m<\gamma_{0}(\ln \ln H)^{1 / 5}$ a precise enough estimate for $L$ is obtained. But for $m \geqslant \gamma_{0}(\ln \ln H)^{1 / 5}$ it becomes rough and the greater is $m$, the more and more rough it becomes. 
Theorem 3 established a precise enough estimate of $\varphi$ for

$$
n^{4 m} \ln (n+1)<\Omega_{0}(m) \ln \ln H,
$$

But $\Omega_{0}(m)$ is an ineffective constant. This estimate becomes not sharp for $n^{4 m} \ln (n+1) \geqslant \Omega_{0}(m) \ln \ln H$. In Theorem 3 the result is effective and a precise estimate takes place for $m(n+1)^{4 m} \ln (n+1) \leqslant \gamma^{-1} \ln \ln H$. The situation is similar in Theorems 4 and 5.

The theorems proved may be applied to many concrete functions. The result can be written down at once if we have already proved either the linear independence or the algebraic independence over $\mathbf{C}(z)$ of the considered functions.

Many works of this kind have been published. The review of some of them one can find in [2]. The theorems of the present work enable us to refine all the estimates published in [3] and [4].

Let us give some simple examples. Let $\alpha_{1}, \ldots, \alpha_{m}$ be distinct numbers from $\mathbf{I}$. It is well known that the functions $e^{\alpha_{1} z}, \ldots, e^{\alpha_{m}}$ are linearly independent over $\mathbf{C}(z)$. They satisfy the system $y_{k}^{\prime}=\alpha_{k} y, k=1, \ldots, m$. It is also evident that these functions form an irreducible set of functions. Thus by Theorem 1 we have

where

$$
L_{\mathrm{I}}\left(e^{\alpha_{1}}, \ldots, e^{\alpha_{m}} ; H\right)>C H^{a},
$$

and

$$
a=1-m-\gamma m^{7 / 2}\left(\ln \ln (H+2)^{-\frac{1}{2}},\right.
$$

where

$$
C= \begin{cases}1, & \text { if } \rho<1, \\ \exp \left\{-(2 m-1) \exp \left(\gamma^{2} m^{5}\right)\right\}, & \text { if } \rho \geqslant 1,\end{cases}
$$

$$
\rho=\gamma m^{5 / 2}(\ln \ln (H+2))^{-\frac{1}{2}} \text {. }
$$

Consider the $E$-functions

$$
K_{\lambda, \mu}(z)=\sum_{n=0}^{\infty} \frac{(-1)^{n}}{(\lambda+1) \ldots(\lambda+n)(\mu+1) \ldots(\mu+n)}\left(\frac{z}{2}\right)^{2 n}, \quad \lambda, \mu \neq-1,2, \ldots
$$

satisfying the differential equation

$$
y^{\prime \prime}+\frac{2 \lambda+2 \mu+1}{z} y^{1}+\left(1+\frac{4 \lambda \mu}{z^{2}}\right) y=\frac{4 \lambda \mu}{z^{2}}
$$

In [11] it is proved that if $\lambda, \mu \in \mathbf{Q}, \lambda-\mu \neq \frac{1}{2}(2 k+1), k \in \mathbf{Z}$, then the $\frac{1}{2}(n+1)(n+2)$ products

$$
K_{\lambda, \mu}^{k_{1}}(z) K_{\lambda, \mu}^{k_{2}}(z), \quad 0 \leqslant k_{1}+k_{2} \leqslant n,
$$

for any $n=1,2, \ldots$, form an irreducible set of functions. Therefore by Theorem $3^{\prime}$ for such $\lambda$ and $\mu$ and for any $\xi \in \mathbf{I}, \xi \neq 0$ the inequality

$$
\varphi_{\mathbf{I}}\left(K_{\lambda, \mu}(\xi), K_{\lambda, \mu}^{\prime}(\xi) ; n ; H\right)>C H^{a}
$$


holds, where

and

$$
a=-\frac{1}{2} n(n+3)-\gamma n^{6}(\ln (n+1))^{\frac{1}{2}}(\ln \ln (H+2))^{-\frac{1}{2}},
$$

where

$$
C= \begin{cases}1, & \text { if } \rho<1, \\ \exp \left\{-\left(n^{2}+3 n+1\right) \exp \left(\gamma^{2} n^{8} \ln (n+1)\right\},\right. & \text { if } \rho \geqslant 1,\end{cases}
$$

$$
\rho=\gamma n^{4}(\ln (n+1))^{\frac{1}{2}}(\ln \ln (H+2))^{-\frac{1}{2}} .
$$

In a similar way one can obtain the quantitative estimates, corresponding to all the theorems proved in [11].

Consider the function $K_{\lambda}(z)=K_{\lambda, 0}(z)$. Let $\xi \in \mathbf{I}, \xi \neq 0$ and $\alpha_{1}, \ldots, \alpha_{m}$ be distinct numbers from $I$. Then, arguing as in [11] it is easy to show that the set of $\frac{1}{2}(n+1)(n+2)$ products

$$
K_{\lambda}^{k_{1}}(\xi z) K_{\lambda}^{k_{2}}(\xi z) \exp \left(\left(\kappa_{1} \alpha_{1}+\ldots+\kappa_{m} \alpha_{m}\right) z\right), \quad 0 \leqslant k_{1}+k_{2}=n, \quad \kappa_{1}+\ldots+\kappa_{m}=1,
$$

form an irreducible set of functions. Then by Theorem $s^{\prime}$ with $s=1, t=1, m_{1}=2$, $\mu_{1}=m$, we have the inequality

$$
\varphi_{\mathrm{I}}\left(K_{\lambda}(\xi), K_{\lambda}^{\prime}(\xi) ; e^{\alpha_{1}}, \ldots, e^{\alpha_{m}} ; n ; 1 ; H\right)>C H^{a}
$$

where

$$
a=1-\frac{1}{2}(n+1)(n+2)-\gamma n^{6} m^{3}(n+\ln (n+1))^{\frac{1}{2}}(\ln \ln (H+2))^{-\frac{1}{2}},
$$

and

$$
C= \begin{cases}1, & \text { if } \rho<1, \\ \exp \left\{-((n+1)(n+2) m-1) \exp \gamma^{2} n^{8} m^{4}(m+\ln (n+1))\right\}^{\frac{1}{2}}, & \text { if } \rho \geqslant 1\end{cases}
$$

where

$$
\rho=\gamma n^{4} m^{2}(m+\ln (n+1))^{\frac{1}{2}}(\ln \ln (H+2))^{-\frac{1}{2}}
$$

or the inequality

$$
\left|P_{1}\left(K_{\lambda}^{\prime}(\xi), K_{\lambda}^{\prime}(\xi)\right) e^{\alpha_{1}}+\ldots+P_{m}\left(K_{\lambda}^{\prime}(\xi), K_{\lambda}^{\prime}(\xi)\right) e^{\alpha_{m}}\right|>C H^{a},
$$

where $a$ is as above, $P_{k}\left(x_{1}, x_{2}\right) \in Z_{1}\left[x_{1}, x_{2}\right]$ and $\operatorname{deg} P_{k} \leqslant n, k=1, \ldots, m$.

Denote

$$
\omega=i \frac{K_{0}(2 i)}{K_{0}^{\prime}(2 i)}=1+\frac{1}{2+\frac{1}{3}+} \cdot \frac{1}{+\frac{1}{n}+} \cdot
$$

Then by Theorem 4

$$
\varphi(\omega ; n ; H)>C H^{b}
$$


where

$$
b=-n-\gamma n^{3}(\ln (n+1))^{\frac{1}{2}}(\ln \ln (H+2))^{-\frac{1}{2}},
$$

and

$$
C= \begin{cases}1, & \text { if } \rho<1, \\ \exp \left\{-(2 n+1) \exp \left(\gamma^{2} n^{4} \ln (n+1)\right)\right\}, & \text { if } \rho \geqslant 1,\end{cases}
$$

where

$$
\rho=\gamma n^{2}(\ln (n+1))^{\frac{1}{2}}(\ln \ln (H+2))^{-\frac{1}{2}} .
$$

In all the examples given, one can calculate the constants $\gamma$ if one makes a more precise calculation of all the constants in the lemmas.

\section{References}

[1] Н. И. Фельдман, А. Б. Шидловский (1967), 'Развитие и современное состояние теории трансцендентных чисел', Успехи матем. наук, 22 (3), 3-81.

[2] А. Б. Шидловскмй (1973), 'Об арифметических свийствах значений аналитических функций', Труды МИАН СССР им. В. А. Стеклова, 132, 169-202.

[3] А. Б. Шидловский (1967), 'Об оценке меры трансцендентности значений Е-функций', Матем. заметки, 2 (1), 33-44.

[4] C. L. Sigel (1929), 'Uber einige Anwendungen Diophantischer Approximationen', Abhand. preuss. Acad. Wiss., $N 1,1-70$.

[5] C. L. Sigel (1949), Transcendental numbers (Princeton) University Press.

[6] А. Б. Шидловский (1977), 'Об оценках меры трансцендентности значений Е-функций', Вестник МГУ, серия математика, механика (6), 3-10.

[7] А. Б. Шидловский (1959), 'О критерии алгебраической независимости значений одного класса целых функций', Изв. АН СССР, серия матем. 23 (1), 35-66.

[8] А. Б. Шидловский (1966), 'К общей теореме об алгебраической независимости значений Е-функций', ДАН СССР, 171 (4), 810-813.

[9] А. Б. Шидловский (1967), 'О6 оценках меры трансцендентности значений Е-функций', Успехи матем. наук, 22 (3), 245-246.

[10] Ю. Б. Нестеренко (1977), 'Оценки порядков нулей функций одного класса и их приложения в теории трансцендентных чисел', Изв. АН СССР, серия матем., 41 (2), 253-284.

[11] А. Б. Шидловский (1959), 'О трансцендентности и алгебраической независимости значений целых функций некоторых классов', Уч. записки МГУ, вып. 186, математика, 9, 11-70.

Department of Mathematics

Moscovskii University

Mehmat, Moscow, 117234

USSR 